\title{
Sexual dimorphism in gastric cancer: tumor-associated neutrophils predict patient outcome only for women
}

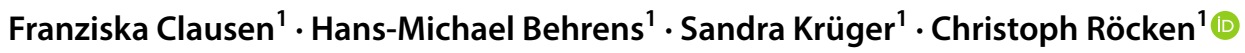

Received: 26 May 2019 / Accepted: 13 November 2019 / Published online: 19 November 2019

(c) The Author(s) 2019

\begin{abstract}
Purpose Tumor-associated neutrophils (TANs) are part of the tumor immune microenvironment (TIME) and may contribute to gastric cancer (GC) biology. We hypothesized that TAN are enriched in the TIME, show sex-specific differences, and correlate with patient outcome.

Methods We analyzed the distribution and putative tumor biological significance of TANs in a well-characterized, therapynaïve, European GC cohort using immunohistochemical staining of myeloperoxidase (MPO), and digital image analysis using Definiens Tissue Studio ${ }^{\circledR}$.

Results Different tumor compartments were examined, and TAN densities were correlated with various clinicopathological patient characteristics. TAN density showed a large interindividual variability ranging from 0 to $6711.0 \mathrm{TANs} / \mathrm{mm}^{2}$. Intratumoral distribution patterns were inhomogeneous (tumor surface vs. tumor center vs. invasion front) and correlated significantly with Laurén phenotype, tumor grade, and microsatellite status in the tumor center and invasion front. In the multivariate analysis, TAN density in the invasion front was an independent predictor of tumor-specific survival only for women $(\mathrm{HR}=2.77, p<0.001)$. In men, no correlation was found between TAN density and survival.

Conclusion With regard to TANs, our study independently validates sexual dimorphism in GC biology.
\end{abstract}

Keywords Gastric cancer · Tumor immune microenvironment $\cdot$ Tumor-associated neutrophils $\cdot$ Sexual dimorphism

\section{Introduction}

Gastric cancer (GC) is the fifth most common cancer worldwide and the third most common cause of cancer death. Incidence and mortality rates are steadily declining, except for cancers of the cardia and gastroesophageal junction, whose incidence has been stable or even increasing. GC is generally more common in men, and incidence increases with patient age (ENCR 2017; Stewart and Wild 2014). Major risk factors are infection with $H$. pylori and dietary habits. In addition, a minority of GC is also linked to Epstein-Barr virus (EBV) infection (Humans 2012). Family history and

Electronic supplementary material The online version of this article (https://doi.org/10.1007/s00432-019-03082-z) contains supplementary material, which is available to authorized users.

Christoph Röcken

christoph.roecken@uksh.de

1 Department of Pathology, Christian-Albrechts-University, Arnold-Heller-Str. 3, Haus U33, 24105 Kiel, Germany gene polymorphisms modulate individual cancer risk (Saeki et al. 2013).

Neutrophils are an essential part of the innate immune system and provide protection against microbial infections. Tumor-associated neutrophils (TANs) can be found as part of the tumor immune microenvironment (TIME) (Binnewies et al. 2018). They are recruited to the tumor site by a chemotactic gradient involving different cytokines, chemokines, and growth factors, provided by the tumor itself or recruited cells. TANs have been associated with a poor prognosis in cancers, most notably in hepatocellular carcinoma, intrahepatic cholangiocarcinoma, head and neck cancer, renal cell carcinoma and non-small-cell lung cancer (Shen et al. 2014). However, a dual role of TANs in cancer biology has been described since they can both promote and inhibit cancer progression. Tumor-suppressing N1-TANs show direct and antibody-dependent cell-mediated cytotoxicity against tumor cells. They also produce proinflammatory cytokines and recruit $\mathrm{CD} 8^{+} \mathrm{T}$ cells (Shaul and Fridlender 2018). N2-TANs are considered tumor promoting. They promote tumor extravasation and angiogenesis, suppress the immune 
system, and recruit $\mathrm{CD} 4^{+} \mathrm{T}$ cells, and so promote tumor growth and metastasis (Shaul and Fridlender 2018). IFN- $\gamma$ might stimulate neutrophils to upregulate programmed cell death 1 ligand 1 (PD-L1) and suppress T cell proliferation. Both N1- and N2-TANs are considered mature neutrophils (Shaul and Fridlender 2018). Factors produced by tumors and stroma have been postulated to influence TAN differentiation (Fridlender et al. 2009; Piccard et al. 2012). Collectively, these data provide evidence that TANs, in general, play an active role in cancer biology.

In the past, a limited number of studies provided evidence that TANs may also be involved in GC biology, showing divergent results with regard to the protective (Abe et al. 2016; Caruso et al. 2002; Huang et al. 2018; Zhang et al. 2018) or promoting effects (Li et al. 2017; Wang et al. 2017; Zhao et al. 2012) of TANs. Only a single study was carried out on Western patients, which, interestingly, showed evidence of a sex-dependent effect (Caruso et al. 2002) pointing towards sexual dimorphism. Indeed, GC shows a striking sex-specific difference in its susceptibility. According to the European Network of Cancer Registries, the estimated GC incidence in men is almost double that of women. A similar picture was observed for mortality, with an estimated 63,600 stomach cancer deaths in men and 43,700 in women (ENCR 2017). These differences cannot be explained by $H$. pylori infection, the major risk factor for GC (Brusselaers et al. 2017; Group 2001). However, the immune response shows sex-specific differences with regard to infectious diseases, vaccination and autoimmunity, and the effector functions of immune cells are influenced by estrogen and androgen exposure (Markle and Fish 2014). This sexual dimorphism in immune response capacity is now well recognized, and differences in immune surveillance competence between men and women may also contribute to the sex effect observed in malignant tumors (Dorak and Karpuzoglu 2012). However, sexual dimorphism in biomedical science is often not specifically addressed and many studies fail to analyze results by sex (Beery and Zucker 2011).

To fill this gap of information, and in order to shed further light on TIME and sex-specific differences in GC, we aimed to test the following hypotheses in an European GC cohort: (a) TANs in GC are enriched in the TIME; (b) TANs show sex-specific differences; and (c) TAN densities correlate with patient outcome.

\section{Materials and methods}

\section{Ethics}

All executed procedures were in accordance with the ethical standards of the responsible committee on human experimentation (institutional and national) and with the Helsinki
Declaration of 1964 and later versions. Ethical approval was obtained from the local ethical review board (D 453/10 and D 468/17). All patient data were pseudonymized prior to study inclusion. All experimental work complied with all mandatory laboratory health and safety procedures.

\section{Study population}

We retrospectively examined all patients who had undergone either total or partial gastrectomy for adenocarcinoma of the stomach or the gastroesophageal junction between 1997 and 2009. Specimens were obtained from the archive of the Institute of Pathology, University Hospital Kiel. The following patient characteristics were retrieved from the electronic database: tumor location, type of surgery, age at diagnosis, sex, tumor type, tumor grade, residual tumor status, tumor size, depth of invasion, number of lymph nodes resected and number of lymph nodes with metastases. Patients were included if a primary adenocarcinoma of the stomach or gastroesophageal junction was histologically confirmed. Patients were excluded if (1) histology identified a tumor type other than adenocarcinoma, (2) patients had undergone a perioperative chemo- or radiotherapy, (3) basic clinicopathological variables (i.e. sex, Laurén phenotype, T-, N-, and M-category, or UICC stage) or (4) more than two of the study compartments (i.e. mucosa, tumor surface, tumor center, and invasion front) were missing (Suppl. Figure 1). Date of patient death was obtained from the "Epidemiological Cancer Registry" of the state of Schleswig-Holstein, Germany. Follow-up data of those patients who were still alive were retrieved from hospital records and general practitioners.

\section{Histology}

Specimens had been fixed in formalin and embedded in paraffin (FFPE). Paraffin sections were stained with hematoxylin and eosin. Tumors were classified according to Laurén (Lauren 1965). The pathological tumor (T), node (N), distant metastasis (M) stage of all study patients was defined according to the 8th edition of the International Union Against Cancer guidelines (Brierley et al. 2016).

\section{Myeloperoxidase immunohistochemistry}

Immunostaining was carried out using the Bondmax automated slide staining system (Leica Microsystems, Wetzlar, Germany) and a rabbit polyclonal anti-human-MPO antibody (dilution 1:2000; Dako, Carpinteria, CA, USA) diluted with Bond Primary Antibody Diluent (Leica, Newcastle, GB). Immunostaining was visualized with the Bond Polymer Refine Detection Kit (Leica Biosystems, Newcastle, GB). Automated antigen retrieval was carried out using Bond 
Epitope Retrieval Solution (Leica Biosystems, Newcastle, GB) at $20 \mathrm{~min}$. Omission of the primary antibody served as a negative control.

\section{Image analysis and virtual microscopy}

Digital images of immunohistochemically stained tissue sections were obtained using a Leica SCN400 microscopic whole-slide scanner (Leica Biosystems, Nussloch, Germany) at its maximum, nominally 40-times magnification. The pixel-to-pixel distance equals $0.25 \mu \mathrm{m}$ in the virtual image. The scanned images were exported from the scanner system as Leica SCN format files. To detect $\mathrm{MPO}^{+}$cells, semiautomatic image analysis with Definiens Tissue Studio (version 3.6.1, Definiens, München, Germany) was performed at 20-times magnification. Software settings were used to vary the programmed outputs and classify the cells wanted. Tissue-background separation distinguishes between tissue and background (auto threshold; multiple tissue pieces; $10,000 \mu^{2}$ minimum tissue size). Nuclei were detected (nucleus detection: 0.1 hematoxylin threshold; $50 \mu \mathrm{m}^{2}$ typical nucleus size), and virtual cell borders were designated (cell simulation: simulation mode $=$ grow from nuclei; $1 \mu \mathrm{m}$ maximum cell growth). Depending on the intensity of brown chromogen (general settings: stain combination $=\mathrm{HC}$ brown chromogen; IHC marker = cytoplasm), the designated cell was classified as a TAN (cell classification: selected feature $=\mathrm{HC}$ marker intensity; measurement in = cell; threshold none/low $=0.5$ ). Analyses were exported as CSV files, which had to be converted in order to be used. The viewer and painting program VMP was used to mark four tumor compartments (Fig. 1).
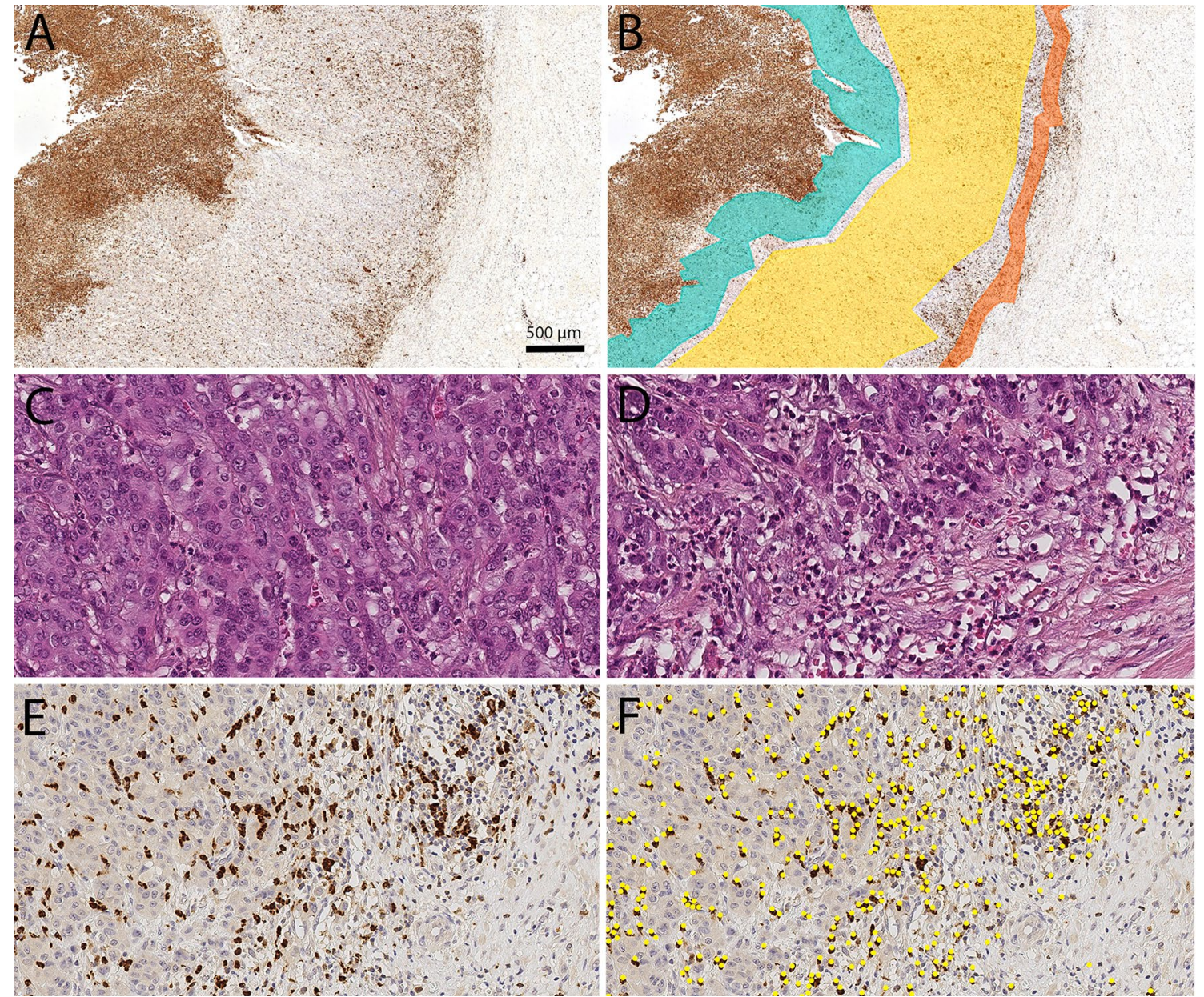

Fig. 1 Digital image analysis: Digital image analysis was used to quantify the spatial distribution of tumor-associated neutrophils (TANs) in gastric cancer. The viewer and painting program VMP was used to mark the tumor compartments: tumor surface (green), tumor center (yellow) and invasion front (orange; a, b). The TAN density was lower in the tumor center $(\mathbf{c})$ than in the invasion front $(\mathbf{d}-\mathbf{f})$.
TAN density was quantified by image analysis using Definiens Tissue Studio ${ }^{\circledR}$ (TANs identified by Definiens are marked as yellow dots; f). All figures were captured from the same tumor. Anti-myeloperoxidase immunostaining $(\mathbf{a}, \mathbf{b}, \mathbf{e}, \mathbf{f})$. Hematoxylin and eosin (c, d). Overview (a, b). Original magnification 400 -fold (c-f) 


\section{Marking compartments}

Four tumor compartments were identified: nonneoplastic peritumoral mucosa, tumor surface, tumor center and invasion front. The peritumoral mucosa consisted of the whole tumor-free area between the muscularis mucosa and the mucin layer. For the tumor surface compartment, we marked an area from the surface up to $500 \mu \mathrm{m}$ into the tumor, avoiding the necrotic layers directly covering the luminal tumor surface. In the tumor center compartment, many parts of the tumor were captured. The marked invasion front was up to $250 \mu \mathrm{m}$ wide and could include small parts of the surrounding stroma. All marked compartments avoided tissuefree space (e.g., artifacts generated during the cutting of the FFPE tissue samples), large necrotic areas or neoplastic glands wider than $200 \mu \mathrm{m}$ filled with neutrophil debris and apoptotic bodies.

\section{Assessment of phenotypic and genotypic characteristics of the study cohort}

H. pylori (Warneke et al. 2013a, b), EBV (Warneke et al. 2013a, b), HER2 (Warneke et al. 2013a, b), MET (Metzger et al. 2016), and MSI (Mathiak et al. 2017) statuses were assessed as previously described.

\section{Study design}

Whole tissue sections from GCs were stained with an antibody directed against MPO. The density of $\mathrm{MPO}^{+} \mathrm{TANs}$ within the tumor compartments, the tumor surface, tumor center, invasion front, and peritumoral area was calculated. The results were correlated with clinicopathological patient characteristics and survival. The study was carried out following REMARK criteria (Altman et al. 2012).

\section{Statistical analysis}

Statistical analyses were performed using SPSS 24.0 and 25.0 (IBM Corporation, New York, USA). TAN densities were first examined as raw score values by the Wilcoxon signed-rank test, then dichotomized at the median and divided into four groups by splitting the densities into quartiles (Q1, Q2, Q3 and Q4). Subsequently, quartiles were grouped into a TAN-low (Q1) and a TAN-high (Q2, Q3, Q4) group. The significance of the correlations between clinicopathological variables was tested using Fisher's exact test. For ordinal scale variables, we used Kendall's tau test for the calculation instead. A p-value was considered statistically significant if $p \leq 0.05$. To compensate false discovery rate within the correlations we applied the Simes (BenjaminiHochberg) procedure (multiple testing correction). Overall (OS) and tumor-specific survival (TSS) were computed using the Kaplan-Meier method and compared by log-rank test to determine the significance of differences between the survival curves. The cohort was also separated by sex, and raw score values, OS, and TSS were calculated again. To estimate their impact, clinicopathological features and genetic alterations were correlated by sex and their impact on survival. Additionally, a multivariate Cox regression model was carried out by counting all factors with $p \leq 0.10$ in the univariate analysis. Sample size calculation for survival analysis was done according to Schoenfeld (1983). We assumed a type II error rate $\beta=0.2$.

\section{Results}

In total, 449 patients fulfilled all study criteria. The median age at the time of diagnosis was 68 years (range 28-92 years). A total of $285(63.5 \%)$ patients were males and $164(36.5 \%)$ were females. $239 \mathrm{GCs}$ had an intestinal phenotype, 136 had a diffuse phenotype, 29 were mixed, and 45 were unclassifiable. Overall survival data were available in $433(96.4 \%)$ cases, and tumor-specific survival data were available in 405 cases $(90.2 \%)$.

\section{TAN density as a function of the tissue compartment}

First, we examined the distribution of TANs in four different compartments, i.e., the peritumoral nonneoplastic mucosa, tumor surface, tumor center, and invasion front (Fig. 1). The median density $\left(\mathrm{n} / \mathrm{mm}^{2}\right)$ of TANs differed significantly between the four different compartments $(p<0.001)$. The highest median density was found at the tumor surface $\left(870.2 \mathrm{TANs} / \mathrm{mm}^{2}\right)$ and the lowest in the nonneoplastic mucosa (57.9 TANs/ $\mathrm{mm}^{2}$; Table 1$)$. Interestingly, the median number of TANs was lower in the tumor center $\left(130.0 \mathrm{TANs} / \mathrm{mm}^{2}\right)$ than in the tumor surface $(870.2$ TANs $/ \mathrm{mm}^{2}$ ) and invasion front (226.8 TANs $/ \mathrm{mm}^{2}$; Table 1$)$. These data provide evidence that TANs in GC are not simply related to an unspecific inflammatory response, e.g., loss of mucosal barrier function, but are specifically spatially enriched at the invasion front.

\section{TAN density as a function of tumor type}

Next, we correlated TAN densities with the histological phenotype according to Laurén. With regard to the individual tumor type (i.e. intestinal, diffuse, mixed, and unclassified), again, median TAN densities varied between the four different compartments (Table 1). In connection with the individual compartments, no significant differences were found in the mucosa and tumor surface. However, TAN densities in the tumor center and invasion front differed significantly between diffuse and non-diffuse type, with GCs having the 


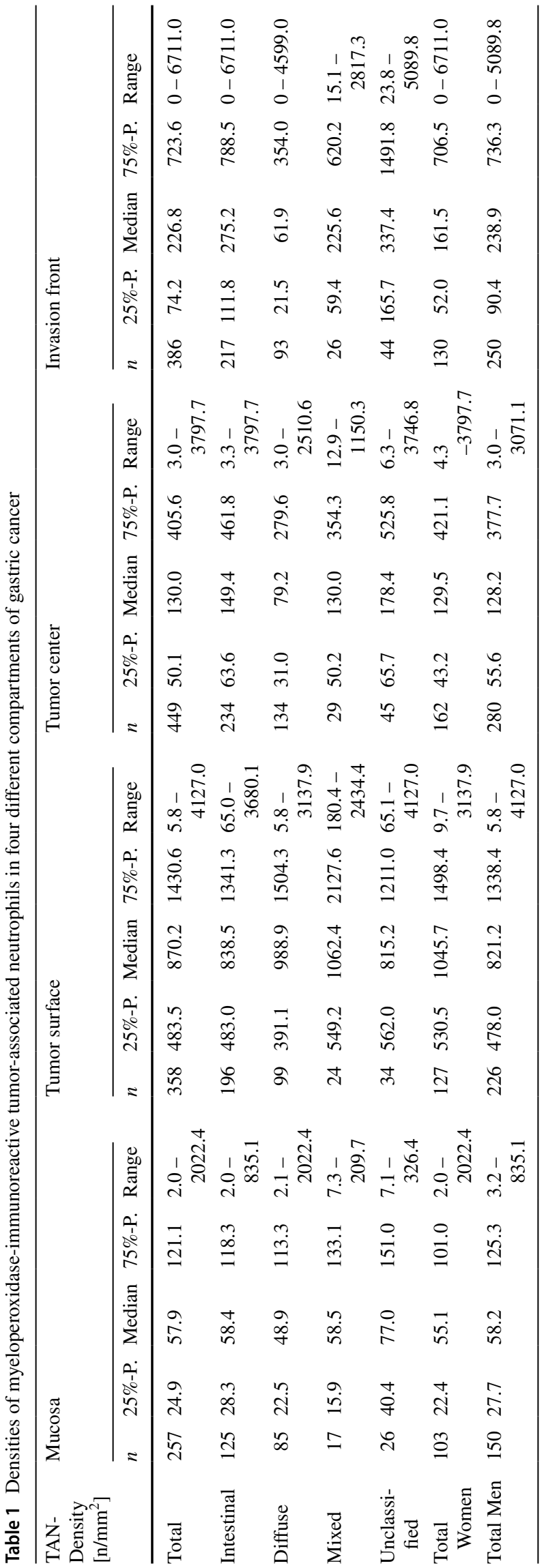

lowest median number of TANs in diffuse type GC (tumor center: $p<0.001$; invasion front: $p<0.001$ ). These data provide evidence that diffuse type GC and non-diffuse type GCs may differ in their ability to recruit TANs into the tumor center and invasion front.

\section{TAN density as a function of sex}

Thereafter, we correlated TAN density with sex. Again, differences were found in the overall distribution of TANs in the four different compartments (Table 1, Fig. 2). However, it was interesting to note that the increase of TAN density from the tumor center to the invasion front was greater in men (128.2 vs. 238.9 TANs $/ \mathrm{mm}^{2}$ ) compared with women (129.5 vs. $161.5 \mathrm{TANs} / \mathrm{mm}^{2}$ ). TANs may contribute to GC biology in both men and women but show subtle spatially related differences in median densities. Clinicopathological features closely linked to sex were: patients' age, localization, Laurén phenotype, EBV- and MET status (Suppl. Table 1).

\section{Exploration of the tumor biological effect of TANs as a function of TAN density}

The assessment of the median values of TANs showed that densities vary, and we next sought to test the hypothesis that TAN density in each compartment (tumor surface, tumor center and invasion front) may correlate with clinicopathological patient characteristics. Using an explorative approach, we first categorized TAN density into "low" and "high" by splitting at the median (Suppl. Table 2). This categorization showed that TAN density correlated with different clinicopathological patient characteristics, e.g., tumor type according to Laurén and MSI status (Suppl. Table 2). However, given the large range of TAN density in GC $\left(0-6711.0 \mathrm{TANs} / \mathrm{mm}^{2}\right)$, we considered that dichotomization of the densities at the median may underestimate the effect of "lower" TAN counts. Therefore, we categorized TAN densities into four quartiles (Q1, Q2, Q3, Q4). This largely showed similar results (Suppl. Table 3), and we then hypothesized that, similar to reference values in whole blood counts, TAN density may have "a (near) physiological range" corresponding to the lowest quartile and a "pathological range" corresponding to the remaining quartiles. Hence, we next dichotomized the densities into a TAN-low group and a TAN-high group by comparing Q1 with Q2-Q4, respectively (Table 2). This showed that TAN density in the tumor center is related to Laurén phenotype (significant after multiple testing correction), tumor grade (significant after multiple testing correction), and EBV and MSI status. At the invasion front, TAN density is related to patient sex, Laurén phenotype (significant after multiple testing correction), tumor grade, T-category, EBV, MSI (significant after 


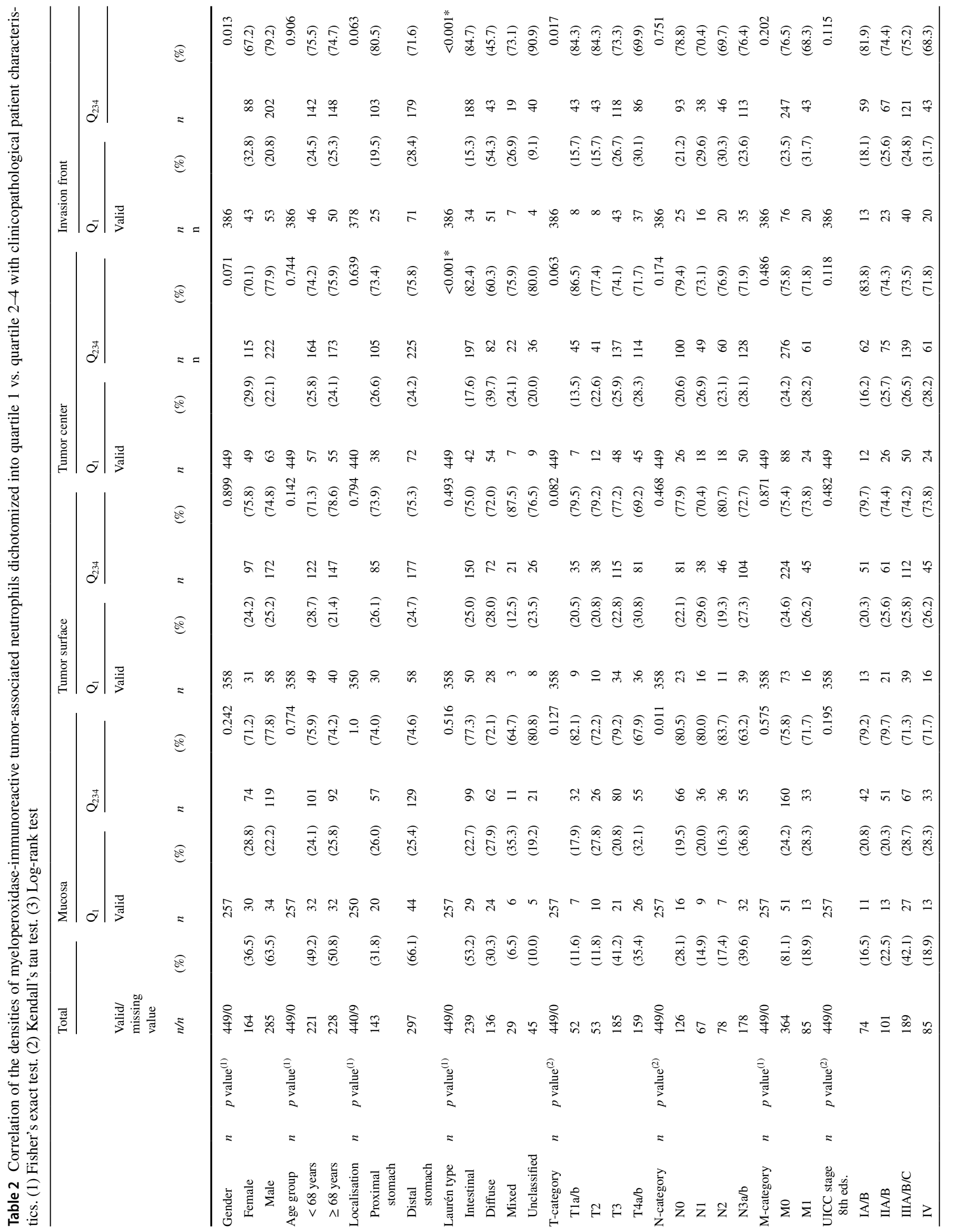




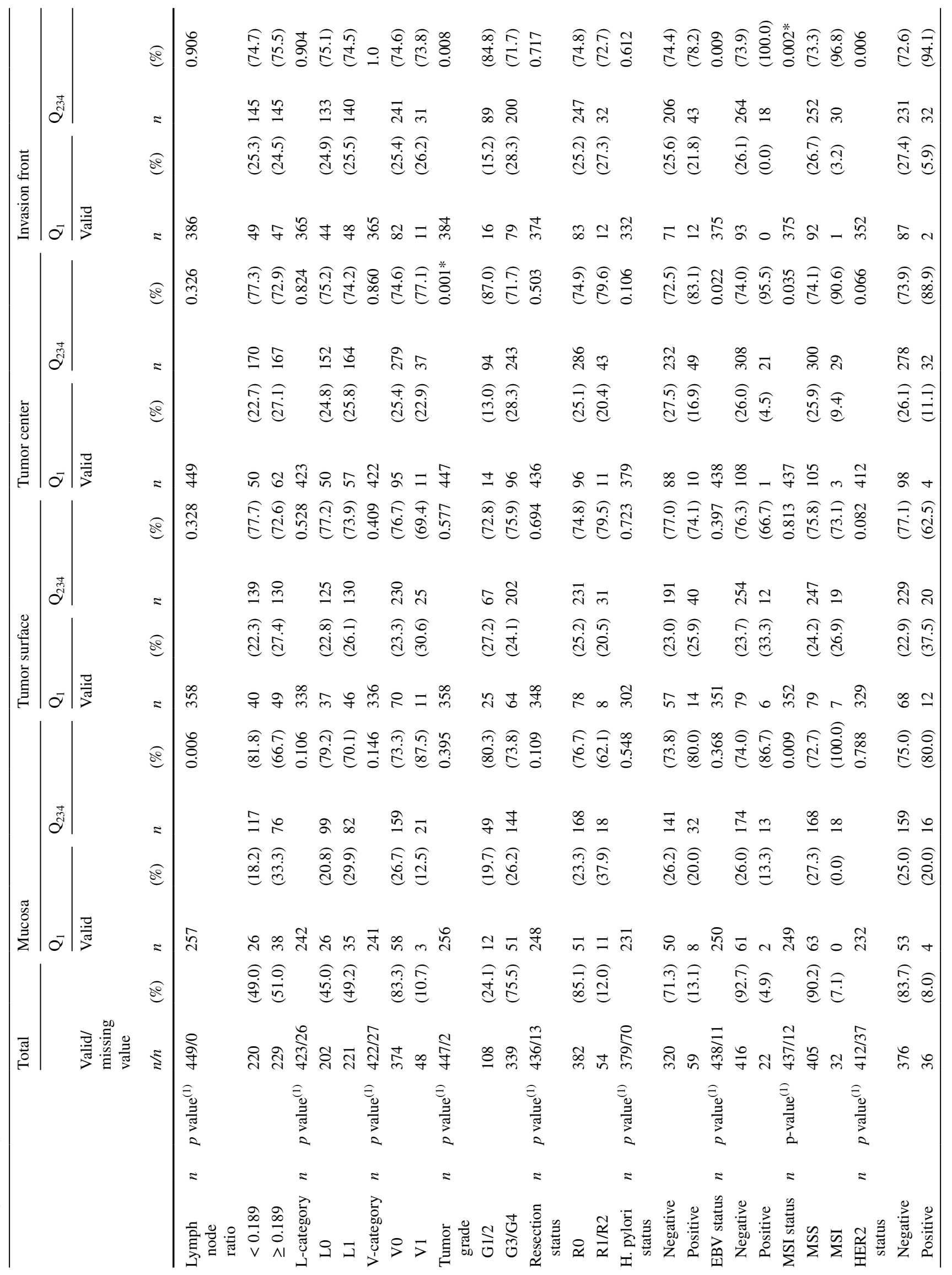




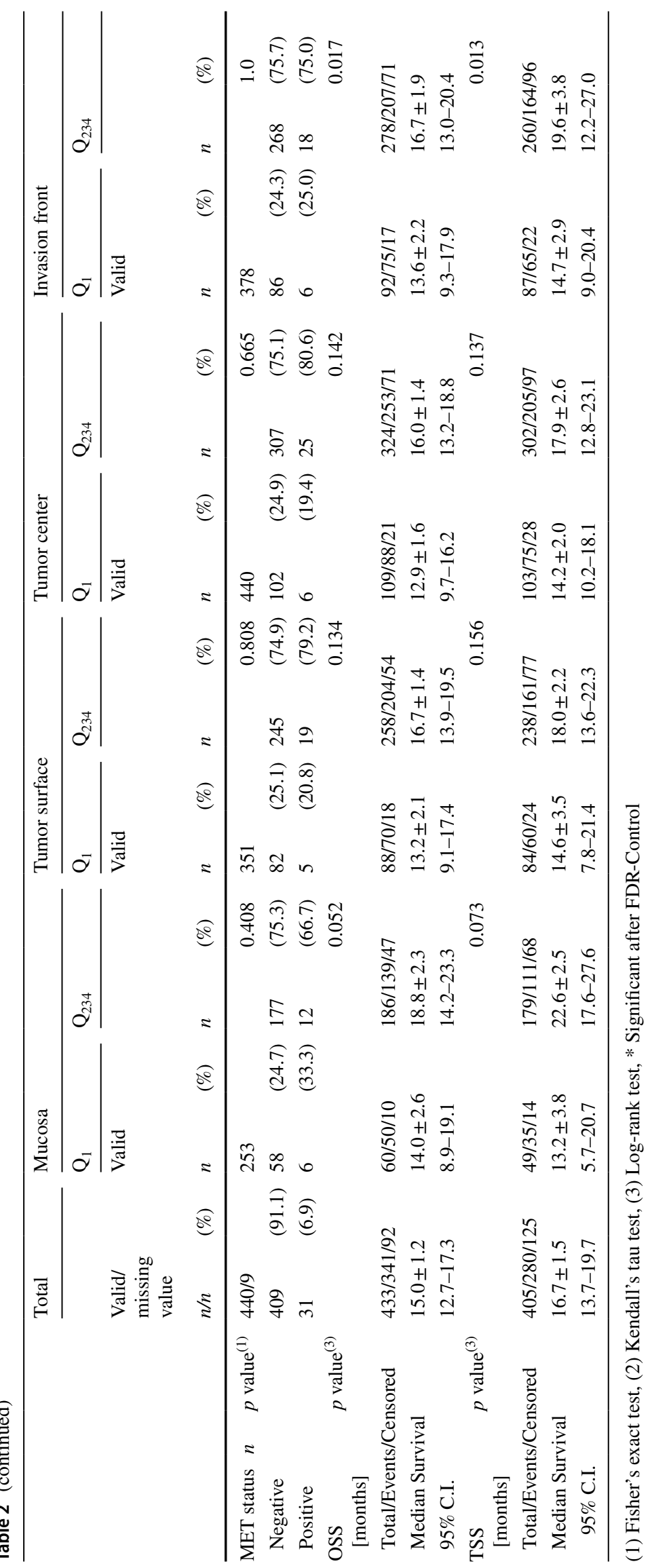


multiple testing correction), and HER2 status and patient survival (Table 2). Collectively, these data show that TANs are biologically relevant to tumors in $\mathrm{GC}$ and that the effect can already be demonstrated when the cutoff is set below the median values (i.e., Q1 vs. Q2-Q4).

\section{Prognostic significance of TAN densities}

Finally, we were interested in exploring the correlation between TAN density and patient survival (Table 2; Suppl. Tables 2 and 3). Dichotomization of the patient cohort at the median (Suppl. Table 2) and at the four quartiles (Suppl. Table 3) showed no differences in tumor-specific survival. However, when TAN density was dichotomized into Q1 (low) and Q2-4 (high), patients with high TAN densities at the invasion front had a much more favorable overall survival (OS; median survival $13.6 \pm 2.2$ months TAN-low vs. $16.7 \pm 1.9$ months TAN-high; $p=0.017)$ and tumor-specific survival (TSS; median survival 14.7 \pm 2.9 months TANlow vs. $19.6 \pm 3.8$ months TAN-high; $p=0.013$ ) compared to patients with low TAN densities. However, after multiple testing correction these results were no longer significant.

Since we noticed sex-specific differences in the TAN density at the invasion front (Table 2), we next explored patient survival separately for men and women. This finally showed that TAN density had no impact on patient survival in men, while it was highly significantly correlated with patient outcome in women. Women with high TAN densities (Q2-Q4) in the tumor center had a better OS (median survival $12.9 \pm 1.6$ months TAN-low vs. $17.9 \pm 2.4$ months TAN-high; $\mathrm{p}=0.079$ ) and a significantly better TSS (median survival $12.8 \pm 1.5$ months TAN-low vs. $18.8 \pm 3.7$ months TAN-high; $p<0.026$ ) compared with women with low TAN densities. Women with high TAN densities (Q2-Q4) at the invasion front had also a significantly better OS (median survival $12.1 \pm 2.2$ months TAN-low vs. $26.3 \pm 7.1$ months TAN-high; $p<0.001$ ) and TSS (median survival $12.1 \pm 1.7$ months TAN-low vs. $36.1 \pm 14.0$ months TANhigh; $p<0.001)$ compared with women with low TAN densities (Q1; Fig. 3; Suppl. Fig. 2). This effect was not related to tumor type according to Laurén (Fig. 4) and there was also no general difference of TSS between men and women in our cohort (Suppl. Fig. 3).

\section{Univariate and multivariate survival analysis}

The entire GC collective showed a median OS of 15.0 months and a median TSS of 16.7 months. Patient prognosis significantly depended on the Laurén phenotype, T-, N-, M-, L-, V- , and R-category, UICC stage, grade, lymph node ratio, MSI status, MET status (data not shown), and TAN density at the invasion front $(p=0.013)$. TSS of women depended on Laurén phenotype, T-, N-, M-, L-,

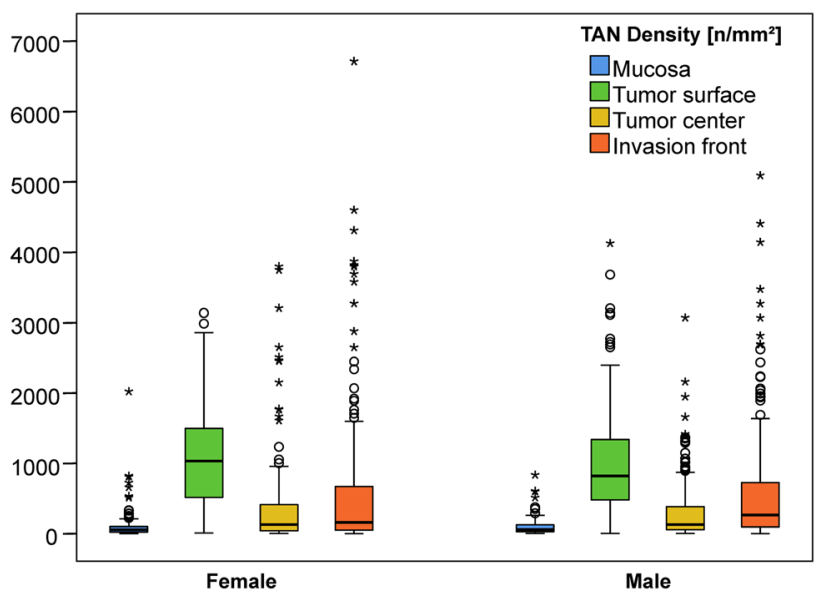

Fig. 2 Boxplots: Densities of myeloperoxidase-immunoreactive tumor-associated neutrophils split by sex in the tumor compartments: nonneoplastic peritumoral mucosa (blue), tumor surface (green), tumor center (yellow) and invasion front (orange)

V-, and R-category, UICC stage, grade, lymph node ratio, MET status, and TAN density at tumor center and invasion front. On multivariate analysis, TAN density at the invasion front (HR 2.77; 95\% CI 1.64-4.77), R-, L-, and N-category turned out to be significant and independent prognosticators of women's tumor-specific survival (Table 3; Suppl. Fig. 1).

\section{Discussion}

Sexual dimorphism in immune response capacity relates to differences in immune surveillance competence between men and women, which may also contribute to the sex effect observed in malignant tumors (Dorak and Karpuzoglu 2012). Our study confirms a previous finding from Caruso et al. (Caruso et al. 2002) of a sex-specific difference in the tumor biological effect of TANs in GC biology by using an independent European patient cohort. The TAN density at the invasion front (HR 2.77; 95\% CI 1.64-4.77) of women with GC was an independent predictor of favorable patient outcome and even surpassed MSI status (Table 3). Interestingly, studies on TANs in Asian cohorts were unable to find any sex-specific differences (Fu et al. 2016; Huang et al. 2018; Li et al. 2017; Zhang et al. 2018; Zhao et al. 2012). However, tumor immunity signatures differ significantly between Asian and non-Asian GC patients, including markers of TANs (i.e., CD66b) and may contribute to differences in clinical outcome (Lin et al. 2015).

Apart from ethnicity, sex has to be considered in regard to the exploitation of immune surveillance and evasion in GC biology, particularly in patients of Western descent. Important immunity genes are carried on the $\mathrm{X}$ chromosome, and sex chromosomes are believed to have a higher impact on 

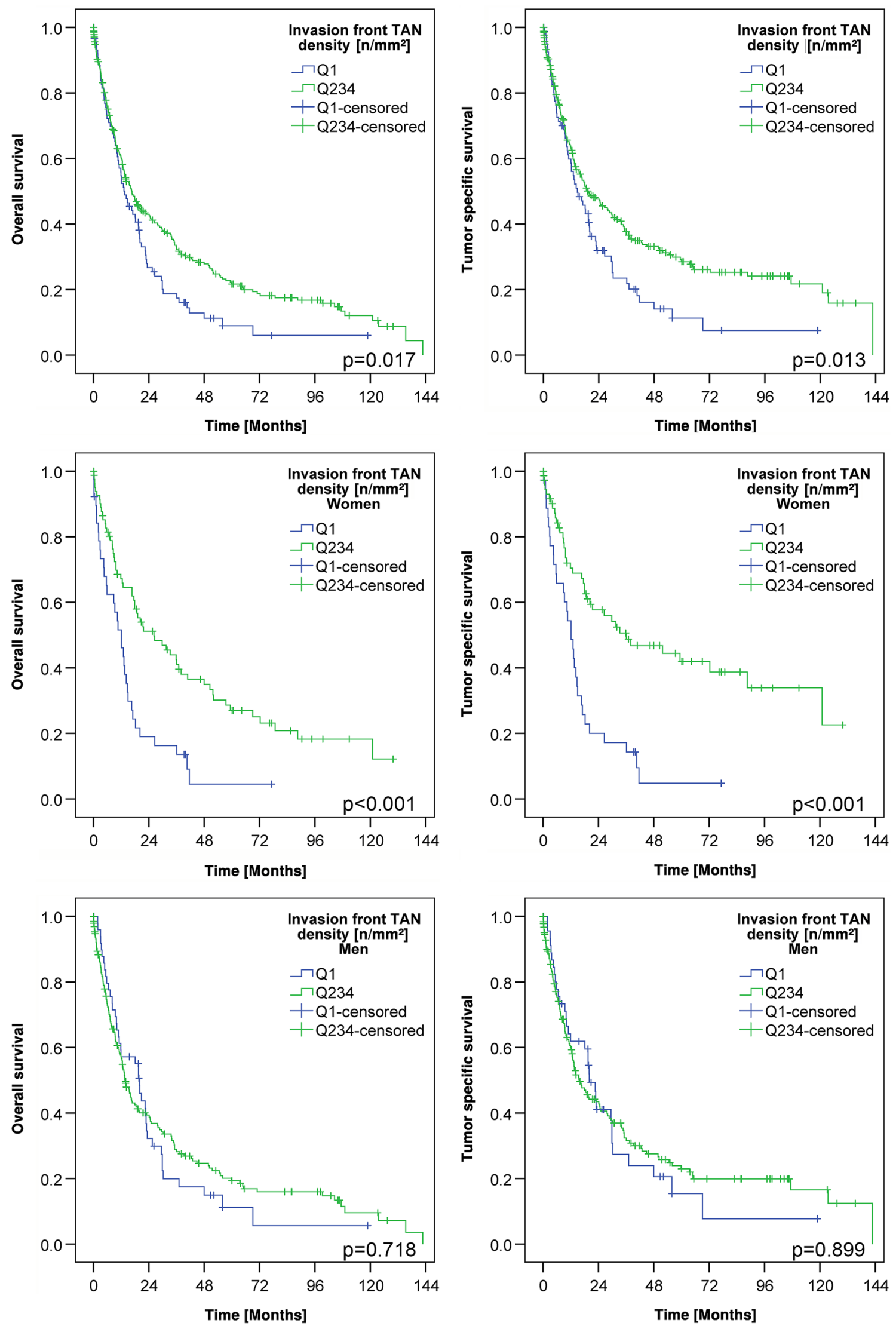
4Fig. 3 Patients' survival: Kaplan-Meier curves of the entire cohort depicting patients' overall and tumor-specific survival according to the densities of myeloperoxidase-immunoreactive tumor-associated neutrophils in the invasion front (dichotomized into quartile 1 vs. quartile 2-4; upper panel). Women with a low density of myeloperoxidase-immunoreactive tumor-associated neutrophils had a poorer survival compared to those with a high density of myeloperoxidaseimmunoreactive tumor-associated neutrophils (middle panel). This effect could not be shown in men (lower panel). All $p$ values shown in the graph were obtained by log-rank test

innate immunity than sex hormones. However, sex hormones regulate the expression of many genes. Female hormones delay neutrophil apoptosis and, depending on the menstrual cycle, modulate their chemotaxis and recruitment (Jaillon et al. 2019). Palli et al. reported that women with a longer fertility duration or a later menopause showed a lower risk of developing GC (Palli et al. 1994). Testosterone, on the other hand, is known to have immunosuppressive effects and causes increased neutrophil activation in noninfectious inflammatory states, and androgen ablation reduces neutrophils (Jaillon et al. 2019). Neutrophils also show sexual differences in appearance (Chatterjee 2014). Spitzer et al. found that their phagocytic response is higher and more resistant to anesthesia and surgery in reproductive female versus male rats (Spitzer and Zhang 1996).

TANs belong to the cellular component of TIME, which had been categorized into three classes (Binnewies et al. 2018): (a) the infiltrated-excluded TIME is characterized by the exclusion of cytotoxic T-cells from the tumor core; (b) the infiltrated-inflamed TIME is considered to be an immunologically 'hot' tumor and is characterized by high infiltration of CTLs expressing programmed cell death protein 1 (PD-1) and leukocytes and tumor cells expressing the immune-dampening PD-1 ligand PD-L1; and (c) a subclass of infiltrated-inflamed TIME, i.e., TLS-TIME, includes tertiary lymphoid structures and lymphoid aggregates whose cellular composition is similar to that found in lymph nodes (Binnewies et al. 2018). TIME is a function of both tumor genotype/phenotype and immunological composition. This might explain the striking differences in TAN densities between diffuse and non-diffuse type GC (Table 1). Recently, a four-tiered molecular classification of GC was proposed, i.e., Epstein-Barr-virus (EBV)-positive, microsatellite instable (MSI), chromosomal instable and genomically stable GCs (Cancer Genome Atlas Research 2014). The diffuse phenotype is often genomically stable and enriched for mutations in $C D H 1, R H O A$ and various other cell adhesion molecules (Cancer Genome Atlas Research 2014). Our findings confirm observations made by Abe et al. (Abe et al. 2016), where TAN densities varied depending on the Laurén phenotype. In contrast, $\mathrm{Fu}$ et al. described different TAN distribution patterns in intestinal and diffuse cancer types, but no differences in TAN densities (Fu et al.
2016). Nonetheless, all these data support the notion that the genotype/phenotype of GC impacts the cellular composition of TIME, including TANs and that the diffuse type GC is characterized by a low density or absence of TANs.

In our cohort, TAN densities were also related to EBV and MSI status, both in tumor center and invasion front. EBV- and MSI-associated GCs are known to induce a strong inflammatory response, which apparently also includes recruitment of TAN, as has also been shown recently by Abe et al. for EBV-GC (Abe et al. 2016). MSI was believed to primarily affect the adaptive immune system. However, innate immunity may also be effective in MSI-GC, and further studies on this topic are warranted.

Neither the tumor nor the TIME is static during tumor progression. This might explain the steady decline of the value of TAN-high (Q2-Q4) at the invasion front. While the number of cases with high TAN density (Q2-Q4) at the invasion front was $84.3 \%$ in T1-tumors, it declined significantly to $69.9 \%$ in T4-tumors (Table 2). Similarly, we previously observed significant differences in the expression of PD-L1 and V-domain Ig suppressor of T cell activation (VISTA) in relation to the T-category (Böger et al. 2016, 2017). Thus, while GC progresses locally, TIME changes with decreasing TAN density, further supporting a putative tumor-protective role of TANs in GC biology. Interestingly, TAN densities were higher in men compared with women, which leads to the conjecture that TANs seem to have different phenotypes in GC: women might be able to activate a more effective N1-TAN phenotype. However, there is currently no validated biomarker for the characterization of TAN phenotypes in tissue sections, and other mechanisms might be effective.

Different from previous attempts on the exploitation of TANs in GC using selected high-power fields (Caruso et al. 2002; Li et al. 2017; Zhao et al. 2012) or tissue micro arrays (Huang et al. 2018), we were specifically interested in the spatial distribution of TANs in GC by using digital image analysis on whole tissue sections and well-defined tumor areas, limiting the risk of observer bias. We found significant differences in the TAN densities with regard to their spatial distribution (tumor surface vs. tumor center vs. invasion front). We noticed a specific enrichment at the invasion front, which was also noticed by $\mathrm{Li}$ et al. (Li et al. 2017). Previously, we already collected ample evidence for the intratumoral heterogeneity of GC, which now also applies to TANs. With regard to the immune evasion mechanism of GC, we previously found different PD-L1 expression patterns in GC (Böger et al. 2016). MSI-GCs were mainly PD-L1-positive at the interface between neoplastic and nonneoplastic tissue, especially in areas of "pushing borders", pointing towards the invasion front as being a specific and tumor-biologically important TIME microniche in GC.

Summing up, our retrospective study on a large European cohort shows that TANs are a specific cellular component of 

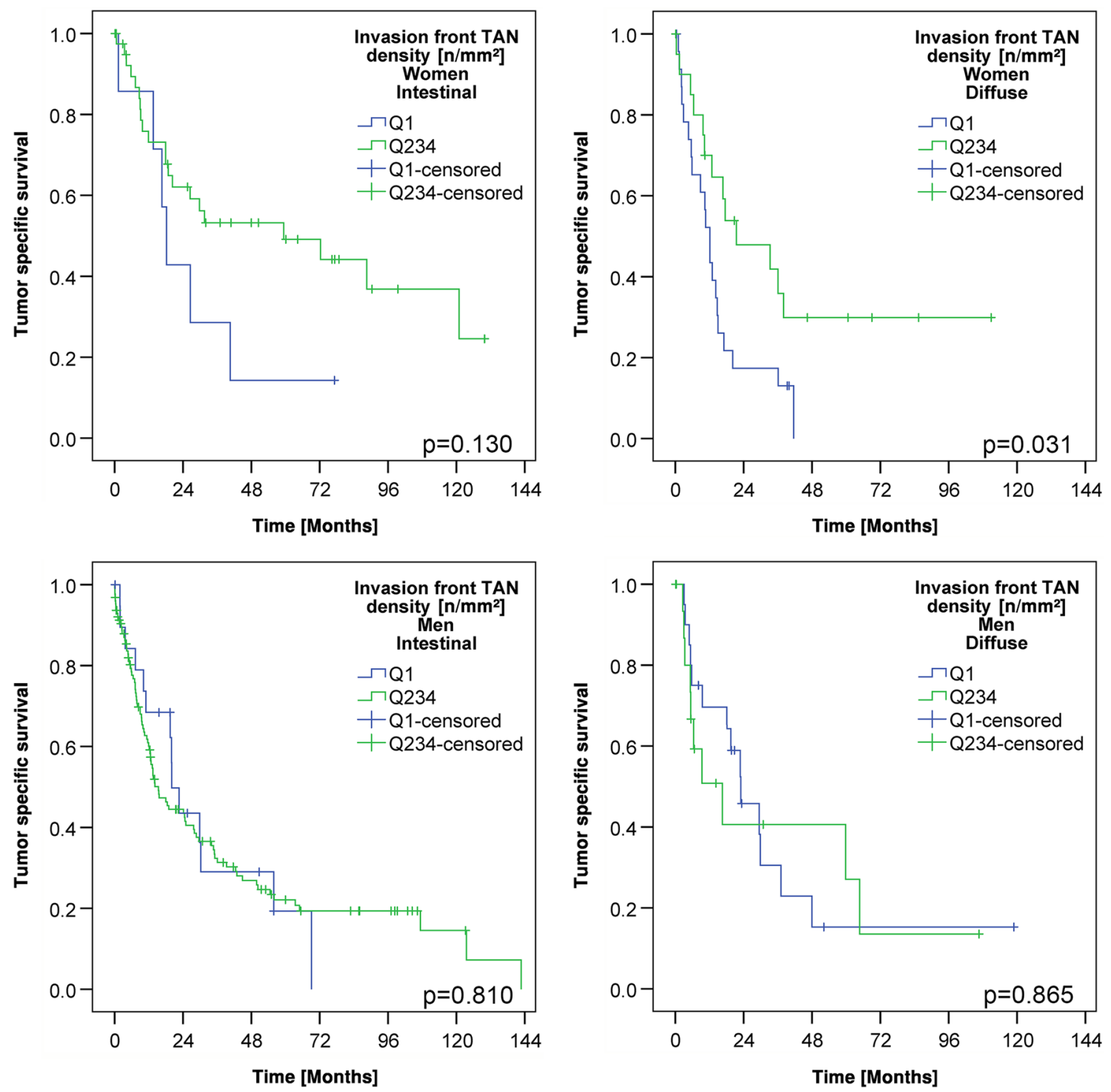

Fig. 4 Patients' survival in intestinal and diffuse phenotype: KaplanMeier curves showing the association between the densities of myeloperoxidase-immunoreactive tumor-associated neutrophils in the invasion front (dichotomized into quartile 1 vs. quartile 2-4) and patients' tumor-specific survival. Independent of the Laurén phenotype, women with a low density of myeloperoxidase-immunoreactive

the TIME with tumor-biological significance. TANs in GC are related to the genotype/phenotype, show unique spatial (tumor surface vs. tumor center vs. invasion front) and "temporal" (T-category) distribution patterns and are even an independent predictor of patient outcome in women. Therefore, our study provides evidence of sexual dimorphism in the immune response capacity to GC, which should be taken into account in future studies and clinical trials.

tumor-associated neutrophils had a poorer survival compared to those with a high density of myeloperoxidase-immunoreactive tumor-associated neutrophils (upper panel). This effect could not be shown in men (lower panel). All $p$ values shown in the graph were obtained by log-rank test

\section{Study limitations}

None of our patients received perioperative or neoadjuvant chemotherapy, and no comment can be made on the effect of chemotherapy on TANs in GC. We also did not study the suitability of endoscopic biopsies to asses TAN densities. However, since the invasion front is rarely captured by biopsies, the assessment of TAN status is prone to a sampling error. Due to the retrospective and observational character of our study using only formalin-fixed and paraffin-embedded tissue samples, we were unable to provide any functional 
Table 3 Univariate and multivariate analysis of tumor specific survival in female gastric cancer patients

\begin{tabular}{|c|c|c|c|c|c|c|c|c|c|}
\hline & & Tumor specifi & ivariate analysis & & & Multivariate an & alysis & & \\
\hline & & $\begin{array}{l}\text { Total/events/ } \\
\text { censored }\end{array}$ & Median survival & $95 \% \mathrm{CI}$ & $p(\log$-rank test $)$ & & HR & $95 \% \mathrm{CI}$ & $p$ value \\
\hline T-category & $\mathrm{T} 1 \mathrm{a} / \mathrm{b}$ & $19 / 3 / 16$ & n.c. & n.c. & $<0.001$ & & & & \\
\hline & $\mathrm{T} 2$ & $15 / 7 / 8$ & $51.7 \pm 23.9$ & 4.9-98.6 & & & & & \\
\hline & $\mathrm{T} 3$ & $55 / 38 / 17$ & $20.3 \pm 8.3$ & $4.1-36.5$ & & & & & \\
\hline & $\mathrm{T} 4 \mathrm{a} / \mathrm{b}$ & $58 / 50 / 8$ & $8.9 \pm 2.1$ & $4.8-13.0$ & & & & & \\
\hline M-category & M0 & $110 / 65 / 45$ & $26.1 \pm 6.3$ & $13.7-38.5$ & $<0.001$ & & & & \\
\hline & M1 & $37 / 33 / 4$ & $7.3 \pm 2.4$ & $2.6-12.0$ & & & & & \\
\hline $\mathrm{N}$-category & N0 & $46 / 17 / 29$ & $88.5 \pm 22.9$ & $43.7-133.4$ & $<0.001$ & & & & 0.003 \\
\hline & $\mathrm{N} 1$ & $25 / 16 / 9$ & $17.1 \pm 3.3$ & $10.5-23.6$ & & N1 vs. N0 & 2.12 & $0.88-5.09$ & 0.094 \\
\hline & $\mathrm{N} 2$ & $20 / 15 / 5$ & $18.2 \pm 4.5$ & $9.4-27.0$ & & N2 vs. N0 & 2.35 & $0.97-5.68$ & 0.058 \\
\hline & $\mathrm{N} 3 \mathrm{a} / \mathrm{b}$ & $56 / 50 / 6$ & $9.3 \pm 1.5$ & $6.3-12.3$ & & N3a/b vs. N0 & 4.07 & $1.95-8.48$ & $<0.001$ \\
\hline L-category & L0 & $69 / 31 / 38$ & $44.6 \pm 16.1$ & $13.1-76.2$ & $<0.001$ & L1 vs. L0 & 2.10 & $1.14-3.85$ & 0.017 \\
\hline & $\mathrm{L} 1$ & $73 / 63 / 10$ & $10.6 \pm 2.7$ & $5.3-16.0$ & & & & & \\
\hline V-category & V0 & $127 / 82 / 45$ & $17.5 \pm 2.2$ & $13.1-21.9$ & 0.030 & & & & \\
\hline & $\mathrm{V} 1$ & $16 / 13 / 3$ & $11.9 \pm 5.8$ & $0.5-23.3$ & & & & & \\
\hline Resection status & R0 & $130 / 81 / 49$ & $18.2 \pm 3.4$ & $11.6-24.8$ & $<0.001$ & R1 vs. R0 & 6.73 & $3.14-14.40$ & $<0.001$ \\
\hline & $\mathrm{R} 1$ & $17 / 17 / 0$ & $5.1 \pm 2.3$ & $0.5-9.7$ & & & & & \\
\hline Tumor grade & $\mathrm{G} 1 / 2$ & $26 / 10 / 16$ & $88.5 \pm 21.5$ & $46.4-130.6$ & $<0.001$ & & & & \\
\hline & $\mathrm{G} 3 / 4$ & $121 / 88 / 33$ & $14.2 \pm 1.9$ & $10.4-17.9$ & & & & & \\
\hline LN-Ratio & $<0.189$ & $79 / 38 / 41$ & $40.6 \pm 13.9$ & $13.2-67.9$ & $<0.001$ & & & & \\
\hline & $\geq 0.189$ & $68 / 60 / 8$ & $9.7 \pm 0.8$ & $8.1-11.3$ & & & & & \\
\hline Laurén type & Intestinal & $56 / 31 / 25$ & $29.8 \pm 13.0$ & $4.3-55.3$ & 0.004 & & & & \\
\hline & Diffuse & $72 / 55 / 17$ & $14.2 \pm 1.6$ & $11.0-17.4$ & & & & & \\
\hline & Mixed & $6 / 5 / 1$ & $4.5 \pm 0.9$ & $2.6-6.4$ & & & & & \\
\hline & Unclassified & $13 / 7 / 6$ & $24.4 \pm 13.2$ & $0-50.2$ & & & & & \\
\hline MSI status & MSS & $134 / 93 / 41$ & $15.5 \pm 1.7$ & $12.1-18.9$ & 0.060 & & & & \\
\hline & MSI & $12 / 5 / 7$ & $51.7 \pm$ n.c. & n.c. & & & & & \\
\hline MET status & Negative & $139 / 92 / 47$ & $17.5 \pm 2.2$ & $13.3-21.7$ & 0.005 & & & & \\
\hline & Positive & $5 / 5 / 0$ & $3.6 \pm 1.3$ & $1.1-6.2$ & & & & & \\
\hline $\begin{array}{l}\text { TAN density in } \\
\text { tumor }\end{array}$ & Q1 & $46 / 36 / 10$ & $12.8 \pm 1.5$ & $9.9-15.8$ & 0.026 & & & & \\
\hline center & Q2-Q4 & $101 / 62 / 39$ & $18.8 \pm 3.7$ & $11.5-26.0$ & & & & & \\
\hline TAN density at & Q1 & $41 / 32 / 9$ & $12.1 \pm 1.7$ & $8.9-15.4$ & $<0.001$ & Q1 vs. Q2-Q4 & 2.77 & $1.64-4.77$ & $<0.001$ \\
\hline invasion front & Q2-Q4 & $75 / 39 / 36$ & $36.1 \pm 14.0$ & $8.6-63.5$ & & & & & \\
\hline
\end{tabular}

data. However, our extensive morphological and statistical analyses may help to generate hypotheses for future experimental studies on the tumor-biological mechanisms of TANs in GC.

Funding None to disclose.

\section{Compliance with ethical standards}

Conflict of interest The authors declare no conflict of interest.

Ethical approval All executed procedures were in accordance with the ethical standards of the responsible committee on human experimen- tation (institutional and national) and with the Helsinki Declaration of 1964 and later versions. Ethical approval was obtained from the local ethical review board (D 453/10 and D 468/17). All patient data were pseudonymized prior to study inclusion. All experimental work complied with all mandatory laboratory health and safety procedures.

Open Access This article is distributed under the terms of the Creative Commons Attribution 4.0 International License (http://creativeco mmons.org/licenses/by/4.0/), which permits unrestricted use, distribution, and reproduction in any medium, provided you give appropriate credit to the original author(s) and the source, provide a link to the Creative Commons license, and indicate if changes were made. 


\section{References}

Abe $\mathrm{H}$ et al (2016) In Epstein-Barr virus-associated gastric carcinoma a high density of CD66b-positive tumor-associated neutrophils is associated with intestinal-type histology and low frequency of lymph node metastasis. Virchows Arch 468:539-548

Altman DG, Mcshane LM, Sauerbrei W, Taube SE (2012) Reporting recommendations for tumor marker prognostic studies (REMARK): explanation and elaboration. PLoS Med 9:e1001216

Beery AK, Zucker I (2011) Sex bias in neuroscience and biomedical research. Neurosci Biobehav Rev 35:565-572

Binnewies $\mathrm{M}$ et al (2018) Understanding the tumor immune microenvironment (TIME) for effective therapy. Nat Med 24:541-550

Böger C et al (2016) PD-L1 is an independent prognostic predictor in gastric cancer of Western patients. Oncotarget 7:24269-24283

Böger C, Behrens HM, Krüger S, Röcken C (2017) The novel negative checkpoint regulator VISTA is expressed in gastric carcinoma and associated with PD-L1/PD-1: a future perspective for a combined gastric cancer therapy? Oncoimmunology 6:e1293215

Brierley JG, Gospodarowicz MK, Wittekind C (2016) TNM classification of malignant tumours, vol 8. Wiley Blackwell, Hoboken

Brusselaers N, Maret-Ouda J, Konings P, El-Serag HB, Lagergren J (2017) Menopausal hormone therapy and the risk of esophageal and gastric cancer. Int J Cancer 140:1693-1699

Cancer Genome Atlas Research N (2014) Comprehensive molecular characterization of gastric adenocarcinoma. Nature 513:202-209

Caruso RA et al (2002) Prognostic value of intratumoral neutrophils in advanced gastric carcinoma in a high-risk area in northern Italy. Mod Pathol 15:831-837

Chatterjee S (2014) Reliability of sexual dimorphism in blood. Indian J Physiol Pharmacol 58:400-402

Dorak MT, Karpuzoglu E (2012) Gender differences in cancer susceptibility: an inadequately addressed issue. Front Genet 3:268

European Network of Cancer Registries (2017) Stomach cancer (SC) fact sheet, https://ec.europa.eu/jrc/en/science-update/new-facts heet-stomach-cancer-europe-released. Accessed 5 Jan 2019

Fridlender ZG et al (2009) Polarization of tumor-associated neutrophil phenotype by TGF-beta: "N1" versus "N2" TAN. Cancer Cell 16:183-194

$\mathrm{Fu} \mathrm{H}$ et al (2016) Persisting and Increasing Neutrophil Infiltration Associates with Gastric Carcinogenesis and E-cadherin Downregulation. Sci Rep 6:29762

Group HaCC (2001) Gastric cancer and Helicobacter pylori: a combined analysis of 12 case control studies nested within prospective cohorts. Gut 49:347-353

Huang X et al (2018) Prognostic significance of the infiltration of CD163(+) macrophages combined with CD66b(+) neutrophils in gastric cancer. Cancer Med 7:1731-1741

IWGOTEOCRT Humans (2012) Biological agents. Volume 100 B. A review of human carcinogens. IARC Monogr Eval Carcinog Risks Hum 100:1-441

Jaillon S, Berthenet K, Garlanda C (2019) Sexual dimorphism in innate immunity. Clin Rev Allergy Immunol 56:308-321
Lauren P (1965) The two histologic main types of gastric carcinoma: diffuse and so-called intestinal-type carcinoma. Acta Pathol Microbiol Scand 64:31-49

Li TJ et al (2017) Interleukin-17-producing neutrophils link inflammatory stimuli to disease progression by promoting angiogenesis in gastric cancer. Clin Cancer Res 23:1575-1585

Lin SJ et al (2015) Signatures of tumour immunity distinguish Asian and non-Asian gastric adenocarcinomas. Gut 64:1721-1731

Markle JG, Fish EN (2014) SeXX matters in immunity. Trends Immunol 35:97-104

Mathiak M et al (2017) Clinicopathologic characteristics of microsatellite instable gastric carcinomas revisited: urgent need for standardization. Appl Immunohistochem Mol Morphol 25:12-24

Metzger ML et al (2016) MET in gastric cancer discarding a 10\% cutoff rule. Histopathology 68(241):253

Palli D et al (1994) Reproductive history and gastric cancer among post-menopausal women. Int J Cancer 56:812-815

Piccard H, Muschel RJ, Opdenakker G (2012) On the dual roles and polarized phenotypes of neutrophils in tumor development and progression. Crit Rev Oncol Hematol 82:296-309

Saeki N, Ono H, Sakamoto H, Yoshida T (2013) Genetic factors related to gastric cancer susceptibility identified using a genome-wide association study. Cancer Sci 104:1-8

Schoenfeld DA (1983) Sample-size formula for the proportional-hazards regression model. Biometrics 39:499-503

Shaul ME, Fridlender ZG (2018) Cancer-related circulating and tumorassociated neutrophils subtypes, sources and function. FEBS J 285:4316-4342

Shen M et al (2014) Tumor-associated neutrophils as a new prognostic factor in cancer: a systematic review and meta-analysis. PLoS One 9:e98259

Spitzer JA, Zhang P (1996) Gender differences in neutrophil function and cytokine-induced neutrophil chemoattractant generation in endotoxic rats. Inflammation 20:485-498

Stewart BW, Wild C (2014) World Cancer Report 2014. WHO Press, Geneva

Wang TT et al (2017) Tumour-activated neutrophils in gastric cancer foster immune suppression and disease progression through GMCSF-PD-L1 pathway. Gut 66:1900-1911

Warneke V et al (2013a) Prognostic and putative predictive biomarkers of gastric cancer for personalized medicine. Diagn Mol Pathol $22: 127-137$

Warneke VS et al (2013b) Her2/neu testing in gastric cancer: evaluating the risk of sampling errors. Ann Oncol 24:725-733

Zhang $\mathrm{H}$ et al (2018) Tumor-infiltrating neutrophils is prognostic and predictive for postoperative adjuvant chemotherapy benefit in patients with gastric cancer. Ann Surg 267:311-318

Zhao JJ et al (2012) The prognostic value of tumor-infiltrating neutrophils in gastric adenocarcinoma after resection. PLoS One 7:e33655

Publisher's Note Springer Nature remains neutral with regard to jurisdictional claims in published maps and institutional affiliations. 